\title{
Red list of vascular plants of the Western Suwalki Lakeland, north-eastern Poland
}

Artur Pliszko

\begin{abstract}
Red list of vascular plants of the Western Suwałki Lakeland, north-eastern Poland. - Acta Mus. Siles. Sci. Natur., 66: 65-73, 2017.

Abstract: The paper presents a regional red list of vascular plant species native to the Western Suwałki Lakeland, north-eastern Poland, based on the IUCN red list categories and criteria. The distribution and abundance data were obtained from the field floristic inventories carried out in 20082016 using the ATPOL cartogram method. The historical occurrences of vascular plant species were revised in the field. The list comprises 203 species, including seven regionally extinct species, 43 critically endangered species, 49 endangered species, 25 vulnerable species, 48 near threatened species, and 31 data deficient species. The results are compared to the Polish national red list of pteridophytes and flowering plants and briefly discussed.
\end{abstract}

Key words: extinction, IUCN red list categories and criteria, threatened species, Poland.

\section{Introduction}

Species extinction is a common phenomenon caused by various natural and anthropogenic factors. The risk of species extinction can be evaluated at global, continental, national or regional level using the uniform categories and criteria recommended by the International Union for Conservation of Nature (IUCN Standards and Petitions Subcommittee 2016). These categories and criteria are widely applied as a standard method for preparing red lists and books of threatened species. In Poland, the first national red list of vascular plants was published in 1986 (Zarzycki 1986) and it has been updated three times (Zarzycki \& Szeląg 1992, 2006, Kaźmierczakowa et al. 2016). At the same time, many regional red lists of plants have been published emphasizing the concerns about nature (e.g. Jakubowska-Gabara \& Kucharski 1999, Głowacki et al. 2003, Kącki et al. 2003, Markowski \& Buliński 2004, Jackowiak et al. 2007).

The Western Suwałki Lakeland (in Polish "Pojezierze Zachodniosuwalskie") is a region in the Polish part of the Lithuanian Lakeland, north-eastern Poland. It covers $830 \mathrm{~km}^{2}$ and is bounded by Błędzianka and Czarna Hańcza rivers on the northern east and by Jarka river on the west (Kondracki 1994). It lies in a transitory temperature climate zone with an influence of continental climate where the mean annual air temperature is about $6.5^{\circ} \mathrm{C}$ and the average annual precipitation is about 550-600 mm (Lorenc 2005). With the main valley of the Rospuda river, the region is characterized by its topographic relief, which is a result of the Vistula Glaciation, showing many early post-glacial landscape forms such as morain hills, sandurs, tunnel valleys and ribbon lakes (Ber 1981). In this rural region, the vegetation is represented mostly by synanthropic and semi-natural plant communities (Stellarietea mediae, Artemisietea vulgaris, Molinio-Arrhenatheretea, and Festuco-Brometea), with the lower share of nemoral forest communities of boreal and subboreal influences (Tilio-Carpinetum, QuercoPinetum, Fraxino-Alnetum, Peucedano-Pinetum, Vaccinio uliginosi-Pinetum, Sphagno girgensohnii-Piceetum, Carici elongatae-Alnetum, Ficario-Ulmetum chrysosplenietosum, and Potentillo albae-Quercetum typicum) (Pliszko 2014 and the literature cited therein). A tentative red list of vascular plants of this region was proposed by Pliszko (2012), however, it was not strictly based on the IUCN red list categories and criteria. The aim of this study is to 
indicate the current risk of extinction for the native vascular plant species occurring in the Western Suwałki Lakeland.

\section{Material and methods}

Field inventories were conducted in 2008-2016, using the ATPOL cartogram method (Zając 1978, Fig. 1). Due to difficulties in the delimitation of the southern boundary of the Western Suwałki Lakeland, the study area was included between North latitude $53^{\circ} 58^{\prime}$ to $54^{\circ} 18^{\prime}$ and East longitude $22^{\circ} 24^{\prime}$ to $22^{\circ} 53^{\prime}$, covering about 740 $\mathrm{km}^{2}$. The distribution and abundance of vascular plant species were studied within 142 square cartogram units of $2.5 \mathrm{~km}$ side (Fig. 2). Moreover, floristic data provided before the $21^{\text {st }}$ century (Abromeit et al. 1898-1940, Zając $\&$ Zając 2001) were revised in the field. The list was arranged in alphabetical order by the scientific names of the taxa following the nomenclature concept by Mirek et al. (2002). The native status of vascular plants in the region followed Zając \& Zając (2001, 2009), Rutkowski (2004) and own observations. The red list was prepared based on the IUCN categories and criteria (IUCN Standards and Petitions Subcommittee 2016), involving regionally extinct (RE), critically endangered (CR), endangered (EN), vulnerable (VU), near threatened (NT) and data deficient (DD) native taxa. The category of least concern (LC) has not been considered in this study due to its lower importance.

\section{Results and discussion}

The red list of vascular plants of the Western Suwałki Lakeland comprises 203 native species (Table 1) which is about $25 \%$ of the indigenous species found in the region (Pliszko 2014a, b, 2015, 2016). Among the listed taxa, there are seven regionally extinct (RE) species, 43 critically endangered (CR) species, 49 endangered (EN) species, 25 vulnerable (VU) species, 48 near threatened (NT) species, and 31 data deficient (DD) species. The list contains 77 species which have been included in Polish red list of pteridophytes and flowering plants (Kaźmierczakowa et al. 2016), however, only seven species have been placed into the same category as in the national list (i.e. Androsace septentrionalis, Eriophorum gracile, Oxytropis pilosa, Potamogeton filiformis, Potentilla rupestris, Utricularia australis, and U. minor). The group of threatened species (CR, EN and VU categories) is $57 \%$ of the listed species. Four species placed into the regionally extinct category, namely Carex brunnescens, C. loliacea, Pedicularis sceptrum-carolinum, and Trifolium spadiceum, have been known only from historical occurrences (Abromeit et al. 1898-1940). Other three species, namely Allium scorodoprasum, Arabis planisiliqua, and Myosurus minimus, became extinct in the past few years. It should be pointed out that the majority of listed species has a higher risk of extinction on the regional scale than on the national scale (e.g. Antennaria dioica, Carex pauciflora, Dactylorhiza majalis, Lathyrus laevigatus, Pulmonaria angustifolia, Senecio congestus, and Trollius europaeus). Some of these species are losing their compact geographic range in north-eastern Poland and the other are threatened due to negative human activities such as deforestation, drainage, chemicalization of agriculture, tourism and recreation. Moreover, there are only 10 species with a lower risk of extinction on the regional scale than on the national scale (e.g. Baeothryon alpinum, Dactylorhiza baltica, Polemonium coeruleum, Potamogeton rutilus, and Viola epipsila). This statement indicates how important for saving some native vascular plants the Western Suwałki Lakeland is. Finally, it is worth to mention that the additional field inventories are needed to establish the extinction risk for the species currently categorized as data deficient. 
Tab 1: Red list of vascular plant species of the Western Suwałki Lakeland.

\begin{tabular}{|c|c|c|c|c|}
\hline \multirow[t]{2}{*}{ No } & \multirow[t]{2}{*}{ Taxon } & \multicolumn{2}{|c|}{$\begin{array}{l}\text { Status in the Western } \\
\text { Suwałki Lakeland }\end{array}$} & \multirow[t]{2}{*}{ Status in Poland* } \\
\hline & & Category & Criterion & \\
\hline 1 & Agrimonia pilosa & $\mathrm{VU}$ & B2a+C2a(i) & NT \\
\hline 2 & Ajuga reptans & NT & - & - \\
\hline 3 & Alchemilla crinita & DD & - & - \\
\hline 4 & A. glabra & DD & - & - \\
\hline 5 & A. plicata & DD & - & - \\
\hline 6 & A. sarmatica & DD & - & - \\
\hline 7 & Alisma gramineum & EN & $\mathrm{B} 2 \mathrm{a}+\mathrm{C} 2 \mathrm{a}(\mathrm{i})$ & VU \\
\hline 8 & A. lanceolatum & EN & $\mathrm{B} 2 \mathrm{a}+\mathrm{C} 2 \mathrm{a}(\mathrm{i})$ & VU \\
\hline 9 & Alliaria petiolata & NT & - & - \\
\hline 10 & Allium scorodoprasum & $\mathrm{RE}$ & - & $\mathrm{VU}$ \\
\hline 11 & A. vineale & $\mathrm{VU}$ & $\mathrm{B} 2 \mathrm{a}+\mathrm{C} 2 \mathrm{a}(\mathrm{i})$ & - \\
\hline 12 & Androsace septentrionalis & VU & $\mathrm{C} 2 \mathrm{a}(\mathrm{i})$ & VU \\
\hline 13 & Anemone sylvestris & NT & - & - \\
\hline 14 & Antennaria dioica & EN & $\mathrm{B} 2 \mathrm{a}+\mathrm{C} 2 \mathrm{a}(\mathrm{i})$ & NT \\
\hline 15 & Anthericum ramosum & NT & - & - \\
\hline 16 & Aquilegia vulgaris & NT & - & - \\
\hline 17 & Arabis planisiliqua & $\mathrm{RE}$ & - & DD \\
\hline 18 & Asperula tinctoria & EN & $\mathrm{B} 2 \mathrm{a}+\mathrm{C} 2 \mathrm{a}(\mathrm{i})$ & VU \\
\hline 19 & Asplenium trichomanes & $\mathrm{CR}$ & $\mathrm{D}$ & - \\
\hline 20 & Astragalus arenarius & VU & $\mathrm{C} 2 \mathrm{a}(\mathrm{i})$ & NT \\
\hline 21 & Astragalus danicus & EN & $\mathrm{B} 2 \mathrm{a}+\mathrm{C} 2 \mathrm{a}(\mathrm{i})$ & VU \\
\hline 22 & Baeothryon alpinum & NT & - & VU \\
\hline 23 & Barbarea stricta & CR & D & - \\
\hline 24 & Batrachium aquatile & EN & $\mathrm{B} 2 \mathrm{a}+\mathrm{C} 2 \mathrm{a}(\mathrm{i})$ & - \\
\hline 25 & Betonica officinalis & VU & $\mathrm{B} 2 \mathrm{a}+\mathrm{C} 2 \mathrm{a}(\mathrm{i})$ & - \\
\hline 26 & Betula humilis & DD & - & EN \\
\hline 27 & Botrychium lunaria & $\mathrm{CR}$ & $\mathrm{D}$ & VU \\
\hline 28 & Brachypodium pinnatum & $\mathrm{CR}$ & $\mathrm{D}$ & - \\
\hline 29 & Bromus benekenii & $\mathrm{CR}$ & $\mathrm{D}$ & - \\
\hline 30 & Butomus umbellatus & $\mathrm{DD}$ & - & - \\
\hline 31 & Calamagrostis stricta & VU & $\mathrm{B} 2 \mathrm{a}+\mathrm{C} 2 \mathrm{a}(\mathrm{i})$ & NT \\
\hline 32 & Callitriche autumnalis & DD & - & DD \\
\hline 33 & Calystegia sepium & DD & - & - \\
\hline 34 & Campanula latifolia & $\mathrm{CR}$ & D & NT \\
\hline 35 & C. rotundifolia & EN & B2a+C2a(i) & - \\
\hline 36 & Cardamine dentata & DD & - & - \\
\hline 37 & Carex atherodes & NT & - & $\mathrm{VU}$ \\
\hline 38 & C. brunnescens & $\mathrm{RE}$ & - & - \\
\hline 39 & C. demissa & $\mathrm{CR}$ & D & NT \\
\hline 40 & C. dioica & EN & $\mathrm{B} 2 \mathrm{a}+\mathrm{C} 2 \mathrm{a}(\mathrm{i})$ & VU \\
\hline 41 & C. disticha & NT & - & - \\
\hline 42 & C. limosa & VU & $\mathrm{B} 2 \mathrm{a}+\mathrm{C} 2 \mathrm{a}(\mathrm{i})$ & NT \\
\hline 43 & C. loliacea & $\mathrm{RE}$ & - & NT \\
\hline 44 & C. montana & EN & $\mathrm{B} 2 \mathrm{a}+\mathrm{C} 2 \mathrm{a}(\mathrm{i})$ & - \\
\hline 45 & C. pauciflora & $\mathrm{CR}$ & $\mathrm{D}$ & EN \\
\hline
\end{tabular}




\begin{tabular}{|c|c|c|c|c|}
\hline 46 & C. pilosa & EN & $\mathrm{D}$ & - \\
\hline 47 & C. praecox & VU & $\mathrm{B} 2 \mathrm{a}+\mathrm{C} 2 \mathrm{a}(\mathrm{i})$ & - \\
\hline 48 & C. riparia & DD & - & - \\
\hline 49 & Catabrosa aquatica & DD & - & VU \\
\hline 50 & $\begin{array}{l}\text { Centaurium erythraea subsp. } \\
\text { erythraea }\end{array}$ & NT & - & - \\
\hline 51 & Cerastium macrocarpum & CR & $\mathrm{D}$ & DD \\
\hline 52 & Ceratophyllum submersum & NT & - & - \\
\hline 53 & Cirsium acaule & $\mathrm{EN}$ & $\mathrm{B} 2 \mathrm{a}+\mathrm{C} 2 \mathrm{a}(\mathrm{i})$ & - \\
\hline 54 & Corallorhiza trifida & $\mathrm{CR}$ & $\mathrm{D}$ & VU \\
\hline 55 & Cornus sanguinea subsp. sanguinea & DD & - & - \\
\hline 56 & Coronilla varia & VU & $\mathrm{C} 2 \mathrm{a}(\mathrm{i})$ & - \\
\hline 57 & Corynephorus canescens & NT & - & - \\
\hline 58 & Crepis succisifolia & NT & - & DD \\
\hline 59 & Cruciata laevipes & EN & $\mathrm{B} 2 \mathrm{a}+\mathrm{C} 2 \mathrm{a}(\mathrm{i})$ & - \\
\hline 60 & Cuscuta epithymum & VU & $\mathrm{B} 2 \mathrm{a}+\mathrm{C} 2 \mathrm{a}(\mathrm{i})$ & - \\
\hline 61 & C. europaea subsp. europaea & DD & - & - \\
\hline 62 & Cyperus fuscus & $\mathrm{CR}$ & $\mathrm{D}$ & - \\
\hline 63 & Cystopteris fragilis & NT & - & - \\
\hline 64 & Dactylis polygama & DD & - & - \\
\hline 65 & Dactylorhiza baltica & NT & - & VU \\
\hline 66 & D. fuchsii & EN & $\mathrm{B} 2 \mathrm{a}+\mathrm{C} 2 \mathrm{a}(\mathrm{i})$ & - \\
\hline 67 & D. maculata & DD & - & - \\
\hline 68 & D. majalis & $\mathrm{EN}$ & $\mathrm{B} 2 \mathrm{a}+\mathrm{C} 2 \mathrm{a}(\mathrm{i})$ & NT \\
\hline 69 & D. ruthei & DD & - & $\mathrm{EN}$ \\
\hline 70 & Dentaria bulbifera & $\mathrm{CR}$ & $\mathrm{D}$ & - \\
\hline 71 & Deschampsia flexuosa & DD & - & - \\
\hline 72 & Dianthus carthusianorum & $\mathrm{EN}$ & $\mathrm{B} 2 \mathrm{a}+\mathrm{C} 2 \mathrm{a}(\mathrm{i})$ & - \\
\hline 73 & Digitalis grandiflora & EN & $\mathrm{B} 2 \mathrm{a}+\mathrm{C} 2 \mathrm{a}(\mathrm{i})$ & - \\
\hline 74 & Draba nemorosa & NT & - & VU \\
\hline 75 & Drosera anglica & $\mathrm{CR}$ & $\mathrm{D}$ & EN \\
\hline 76 & Eleocharis acicularis & DD & - & - \\
\hline 77 & E. quinqueflora & $\mathrm{EN}$ & $\mathrm{B} 2 \mathrm{a}+\mathrm{C} 2 \mathrm{a}(\mathrm{i})$ & VU \\
\hline 78 & Empetrum nigrum & NT & - & - \\
\hline 79 & Epipactis helleborine & $\mathrm{CR}$ & $\mathrm{D}$ & - \\
\hline 80 & E. palustris & NT & - & NT \\
\hline 81 & Equisetum variegatum & DD & - & - \\
\hline 82 & Eriophorum gracile & EN & $\mathrm{B} 2 \mathrm{a}+\mathrm{C} 2 \mathrm{a}(\mathrm{i})$ & EN \\
\hline 83 & E. latifolium & NT & - & - \\
\hline 84 & Euphrasia nemorosa & EN & $\mathrm{B} 2 \mathrm{a}+\mathrm{C} 2 \mathrm{a}(\mathrm{i})$ & - \\
\hline 85 & Filago minima & EN & $\mathrm{B} 2 \mathrm{a}+\mathrm{C} 2 \mathrm{a}(\mathrm{i})$ & - \\
\hline 86 & Filipendula vulgaris & NT & - & - \\
\hline 87 & Fragaria viridis & NT & - & - \\
\hline 88 & Gagea minima & VU & $\mathrm{B} 2 \mathrm{a}+\mathrm{C} 2 \mathrm{a}(\mathrm{i})$ & - \\
\hline 89 & Galeopsis pubescens & $\mathrm{EN}$ & $\mathrm{B} 2 \mathrm{a}+\mathrm{C} 2 \mathrm{a}(\mathrm{i})$ & - \\
\hline 90 & Galium boreale & NT & - & - \\
\hline 91 & Geranium sanguineum & NT & - & - \\
\hline 92 & G. sylvaticum & $\mathrm{EN}$ & $\mathrm{B} 2 \mathrm{a}+\mathrm{C} 2 \mathrm{a}(\mathrm{i})$ & NT \\
\hline 93 & Geum aleppicum & VU & $\mathrm{B} 2 \mathrm{a}+\mathrm{C} 2 \mathrm{a}(\mathrm{i})$ & - \\
\hline 94 & Glyceria nemoralis & EN & $\mathrm{B} 2 \mathrm{a}+\mathrm{C} 2 \mathrm{a}(\mathrm{i})$ & - \\
\hline
\end{tabular}




\begin{tabular}{|c|c|c|c|c|}
\hline 95 & Goodyera repens & EN & $\mathrm{B} 2 \mathrm{a}+\mathrm{C} 2 \mathrm{a}(\mathrm{i})$ & NT \\
\hline 96 & Gypsophila muralis & VU & $\mathrm{B} 2 \mathrm{a}+\mathrm{C} 2 \mathrm{a}(\mathrm{i})$ & - \\
\hline 97 & $\begin{array}{l}\text { Helianthemum nummularium subsp. } \\
\text { obscurum }\end{array}$ & NT & - & - \\
\hline 98 & Hieracium caespitosum & EN & $\mathrm{B} 2 \mathrm{a}+\mathrm{C} 2 \mathrm{a}(\mathrm{i})$ & - \\
\hline 99 & H. cymosum & CR & $\mathrm{D}$ & - \\
\hline 100 & H. sabaudum & CR & $\mathrm{D}$ & - \\
\hline 101 & Hippuris vulgaris & NT & - & - \\
\hline 102 & Holosteum umbellatum & $\mathrm{CR}$ & $\mathrm{D}$ & - \\
\hline 103 & Hypericum montanum & $\mathrm{CR}$ & $\mathrm{D}$ & - \\
\hline 104 & Inula salicina & EN & $\mathrm{B} 2 \mathrm{a}+\mathrm{C} 2 \mathrm{a}(\mathrm{i})$ & - \\
\hline 105 & Juncus alpino-articulatus & VU & $\mathrm{B} 2 \mathrm{a}+\mathrm{C} 2 \mathrm{a}(\mathrm{i})$ & NT \\
\hline 106 & J. filiformis & VU & $\mathrm{B} 2 \mathrm{a}+\mathrm{C} 2 \mathrm{a}(\mathrm{i})$ & NT \\
\hline 107 & Koeleria macrantha & $\mathrm{CR}$ & $\mathrm{D}$ & - \\
\hline 108 & Lamium maculatum & NT & - & - \\
\hline 109 & Laserpitium latifolium & VU & $\mathrm{B} 2 \mathrm{a}+\mathrm{C} 2 \mathrm{a}(\mathrm{i})$ & - \\
\hline 110 & $\begin{array}{l}\text { Lathraea squamaria subsp. } \\
\text { squamaria }\end{array}$ & NT & - & - \\
\hline 111 & Lathyrus laevigatus & EN & $\mathrm{B} 2 \mathrm{a}+\mathrm{C} 2 \mathrm{a}(\mathrm{i})$ & NT \\
\hline 112 & L. niger & VU & $\mathrm{C} 2 \mathrm{a}(\mathrm{i})$ & - \\
\hline 113 & L. palustris & CR & $\mathrm{D}$ & - \\
\hline 114 & Leersia oryzoides & DD & - & NT \\
\hline 115 & Lilium martagon & $\mathrm{EN}$ & $\mathrm{B} 2 \mathrm{a}+\mathrm{C} 2 \mathrm{a}(\mathrm{i})$ & - \\
\hline 116 & Liparis loeselii & $\mathrm{EN}$ & $\mathrm{B} 2 \mathrm{a}+\mathrm{C} 2 \mathrm{a}(\mathrm{i})$ & VU \\
\hline 117 & Listera ovata & EN & $\mathrm{B} 2 \mathrm{a}+\mathrm{C} 2 \mathrm{a}(\mathrm{i})$ & - \\
\hline 118 & Lithospermum officinale & EN & $\mathrm{D}$ & - \\
\hline 119 & Luzula pallescens & EN & $\mathrm{B} 2 \mathrm{a}+\mathrm{C} 2 \mathrm{a}(\mathrm{i})$ & - \\
\hline 120 & Lycopodium clavatum & NT & - & - \\
\hline 121 & Malaxis monophyllos & $\mathrm{CR}$ & $\mathrm{D}$ & VU \\
\hline 122 & Malus sylvestris & $\mathrm{CR}$ & $\mathrm{D}$ & - \\
\hline 123 & Melandrium rubrum & NT & - & - \\
\hline 124 & Mentha longifolia & EN & $\mathrm{B} 2 \mathrm{a}+\mathrm{C} 2 \mathrm{a}(\mathrm{i})$ & - \\
\hline 125 & Mercurialis perennis & NT & - & - \\
\hline 126 & Monotropa hypophegea & $\mathrm{CR}$ & $\mathrm{D}$ & DD \\
\hline 127 & Myosurus minimus & RE & - & - \\
\hline 128 & Myriophyllum verticillatum & DD & - & - \\
\hline 129 & Neottia nidus-avis & NT & - & - \\
\hline 130 & Nuphar pumila & DD & - & VU \\
\hline 131 & Ophioglossum vulgatum & $\mathrm{CR}$ & $\mathrm{D}$ & VU \\
\hline 132 & Orchis mascula subsp. signifera & CR & $\mathrm{D}$ & NT \\
\hline 133 & Oxytropis pilosa & VU & $\mathrm{B} 2 \mathrm{a}+\mathrm{C} 2 \mathrm{a}(\mathrm{i})$ & VU \\
\hline 134 & Parnassia palustris & NT & - & VU \\
\hline 135 & Pedicularis palustris & EN & $\mathrm{B} 2 \mathrm{a}+\mathrm{C} 2 \mathrm{a}(\mathrm{i})$ & VU \\
\hline 136 & P. sceptrum-carolinum & RE & - & - \\
\hline 137 & Petasites spurius & $\mathrm{CR}$ & $\mathrm{D}$ & - \\
\hline 138 & Phegopteris connectilis & $\mathrm{CR}$ & $\mathrm{D}$ & - \\
\hline 139 & Pimpinella major & $\mathrm{CR}$ & $\mathrm{D}$ & - \\
\hline 140 & Platanthera chlorantha & VU & $\mathrm{B} 2 \mathrm{a}+\mathrm{C} 2 \mathrm{a}(\mathrm{i})$ & NT \\
\hline 141 & Polemonium coeruleum & NT & - & VU \\
\hline 142 & Polygala amarella & $\mathrm{CR}$ & $\mathrm{D}$ & - \\
\hline
\end{tabular}




\begin{tabular}{|c|c|c|c|c|}
\hline 143 & Polygonatum odoratum & EN & $\mathrm{B} 2 \mathrm{a}+\mathrm{C} 2 \mathrm{a}(\mathrm{i})$ & - \\
\hline 144 & P. verticillatum & VU & $\mathrm{B} 2 \mathrm{a}+\mathrm{C} 2 \mathrm{a}(\mathrm{i})$ & - \\
\hline 145 & Polygonum mite & DD & - & - \\
\hline 146 & Polypodium vulgare & NT & - & - \\
\hline 147 & Potamogeton acutifolius & VU & $\mathrm{B} 2 \mathrm{a}+\mathrm{C} 2 \mathrm{a}(\mathrm{i})$ & NT \\
\hline 148 & P. alpinus & NT & - & VU \\
\hline 149 & P. filiformis & CR & $\mathrm{D}$ & $\mathrm{CR}$ \\
\hline 150 & P. gramineus & $\mathrm{EN}$ & $\mathrm{B} 2 \mathrm{a}+\mathrm{C} 2 \mathrm{a}(\mathrm{i})$ & VU \\
\hline 151 & P. obtusifolius & CR & $\mathrm{D}$ & NT \\
\hline 152 & P. pusillus & DD & - & NT \\
\hline 153 & P. rutilus & NT & - & CR \\
\hline 154 & P. trichoides & $\mathrm{EN}$ & $\mathrm{D}$ & NT \\
\hline 155 & Potentilla anglica & DD & - & - \\
\hline 156 & P. collina & DD & - & - \\
\hline 157 & P. neumanniana & $\mathrm{CR}$ & $\mathrm{D}$ & - \\
\hline 158 & P. norvegica & $\mathrm{EN}$ & $\mathrm{B} 2 \mathrm{a}+\mathrm{C} 2 \mathrm{a}(\mathrm{i})$ & - \\
\hline 159 & P. reptans & $\mathrm{EN}$ & $\mathrm{B} 2 \mathrm{a}+\mathrm{C} 2 \mathrm{a}(\mathrm{i})$ & - \\
\hline 160 & P. rupestris & $\mathrm{EN}$ & $\mathrm{B} 2 \mathrm{a}+\mathrm{D}$ & $\mathrm{EN}$ \\
\hline 161 & Prunus spinosa & DD & - & - \\
\hline 162 & Pulmonaria angustifolia & $\mathrm{CR}$ & $\mathrm{D}$ & VU \\
\hline 163 & Ranunculus cassubicus & NT & - & - \\
\hline 164 & R. polyanthemos & VU & $\mathrm{B} 2 \mathrm{a}+\mathrm{C} 2 \mathrm{a}(\mathrm{i})$ & - \\
\hline 165 & Rumex aquaticus & DD & - & - \\
\hline 166 & R. maritimus & $\mathrm{EN}$ & $\mathrm{B} 2 \mathrm{a}+\mathrm{C} 2 \mathrm{a}(\mathrm{i})$ & - \\
\hline 167 & Sagina nodosa & VU & $\mathrm{B} 2 \mathrm{a}+\mathrm{C} 2 \mathrm{a}(\mathrm{i})$ & - \\
\hline 168 & Salix starkeana & $\mathrm{CR}$ & $\mathrm{D}$ & VU \\
\hline 169 & Sanguisorba muricata & EN & $\mathrm{B} 2 \mathrm{a}+\mathrm{C} 2 \mathrm{a}(\mathrm{i})$ & DD \\
\hline 170 & S. officinalis & $\mathrm{CR}$ & $\mathrm{D}$ & - \\
\hline 171 & Sanicula europaea & NT & - & - \\
\hline 172 & Saxifraga tridactylites & NT & - & - \\
\hline 173 & Scheuzeria palustris & EN & $\mathrm{B} 2 \mathrm{a}+\mathrm{C} 2 \mathrm{a}(\mathrm{i})$ & VU \\
\hline 174 & Scleranthus perennis & NT & - & - \\
\hline 175 & Scorzonera humilis & NT & - & - \\
\hline 176 & Selinum carvifolia & VU & $\mathrm{B} 2 \mathrm{a}+\mathrm{C} 2 \mathrm{a}(\mathrm{i})$ & - \\
\hline 177 & Senecio congestus & EN & $\mathrm{B} 2 \mathrm{a}+\mathrm{C} 2 \mathrm{a}(\mathrm{i})$ & NT \\
\hline 178 & S. paludosus & NT & - & - \\
\hline 179 & S. sylvaticus & $\mathrm{CR}$ & $\mathrm{D}$ & - \\
\hline 180 & Serratula tinctoria & EN & $\mathrm{B} 2 \mathrm{a}+\mathrm{C} 2 \mathrm{a}(\mathrm{i})$ & - \\
\hline 181 & Spergularia rubra & NT & - & - \\
\hline 182 & Stellaria crassifolia & $\mathrm{CR}$ & $\mathrm{D}$ & VU \\
\hline 183 & S. longifolia & $\mathrm{CR}$ & $\mathrm{D}$ & - \\
\hline 184 & S. uliginosa & DD & - & - \\
\hline 185 & Thalictrum minus subsp. minus & $\mathrm{CR}$ & $\mathrm{D}$ & - \\
\hline 186 & T. simplex & NT & - & VU \\
\hline 187 & Thesium ebracteatum & $\mathrm{EN}$ & $\mathrm{D}$ & VU \\
\hline 188 & Tragopogon orientalis & DD & - & - \\
\hline 189 & Trifolium rubens & $\mathrm{EN}$ & $\mathrm{B} 2 \mathrm{a}+\mathrm{C} 2 \mathrm{a}(\mathrm{i})$ & VU \\
\hline 190 & T. spadiceum & RE & - & DD \\
\hline 191 & Trisetum sibiricum & CR & $\mathrm{D}$ & NT \\
\hline 192 & Trollius europaeus & CR & $\mathrm{D}$ & VU \\
\hline
\end{tabular}




\begin{tabular}{lllll}
\hline $\mathbf{1 9 3}$ & Utricularia australis & NT & - & NT \\
$\mathbf{1 9 4}$ & Utricularia intermedia & EN & B2a+C2a(i) & VU \\
$\mathbf{1 9 5}$ & U. minor & NT & - & NT \\
$\mathbf{1 9 6}$ & V. spicata subsp. spicata & CR & D & - \\
$\mathbf{1 9 7}$ & Vicia tenuifolia & NT & - & - \\
$\mathbf{1 9 8}$ & Vincetoxicum hirundinaria & NT & - & - \\
$\mathbf{1 9 9}$ & Viola epipsila & VU & B2a+C2a(i) & EN \\
$\mathbf{2 0 0}$ & V. hirta & CR & D & - \\
$\mathbf{2 0 1}$ & V. stagnina & EN & B2a+C2a(i) & VU \\
$\mathbf{2 0 2}$ & V. tricolor & DD & - & - \\
$\mathbf{2 0 3}$ & Viscaria vulgaris & NT & - & - \\
\hline
\end{tabular}

* - according to Kaźmierczakowa et al. (2016).

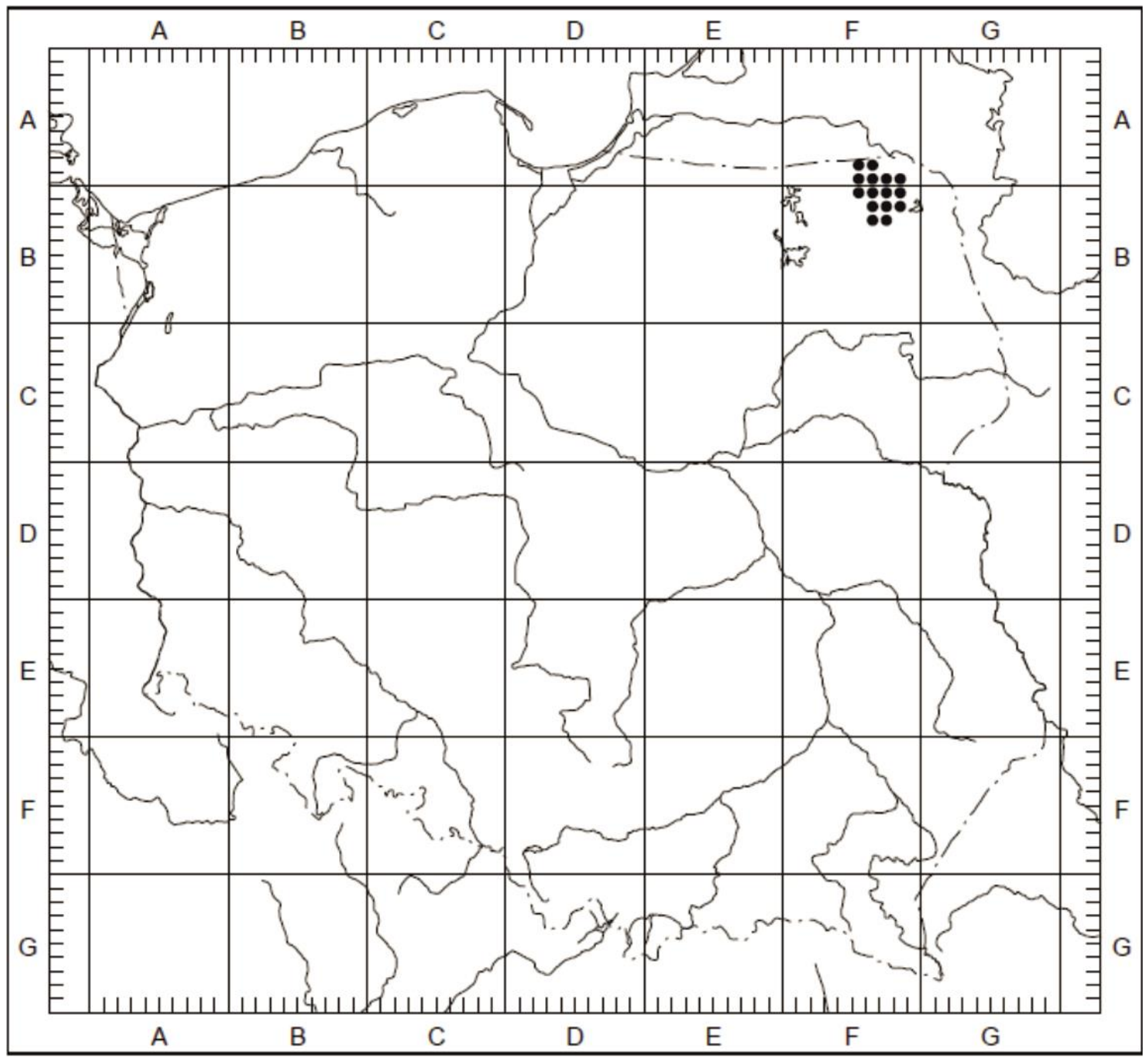

Fig 1: Localization of the study area within the ATPOL cartogram grid. 


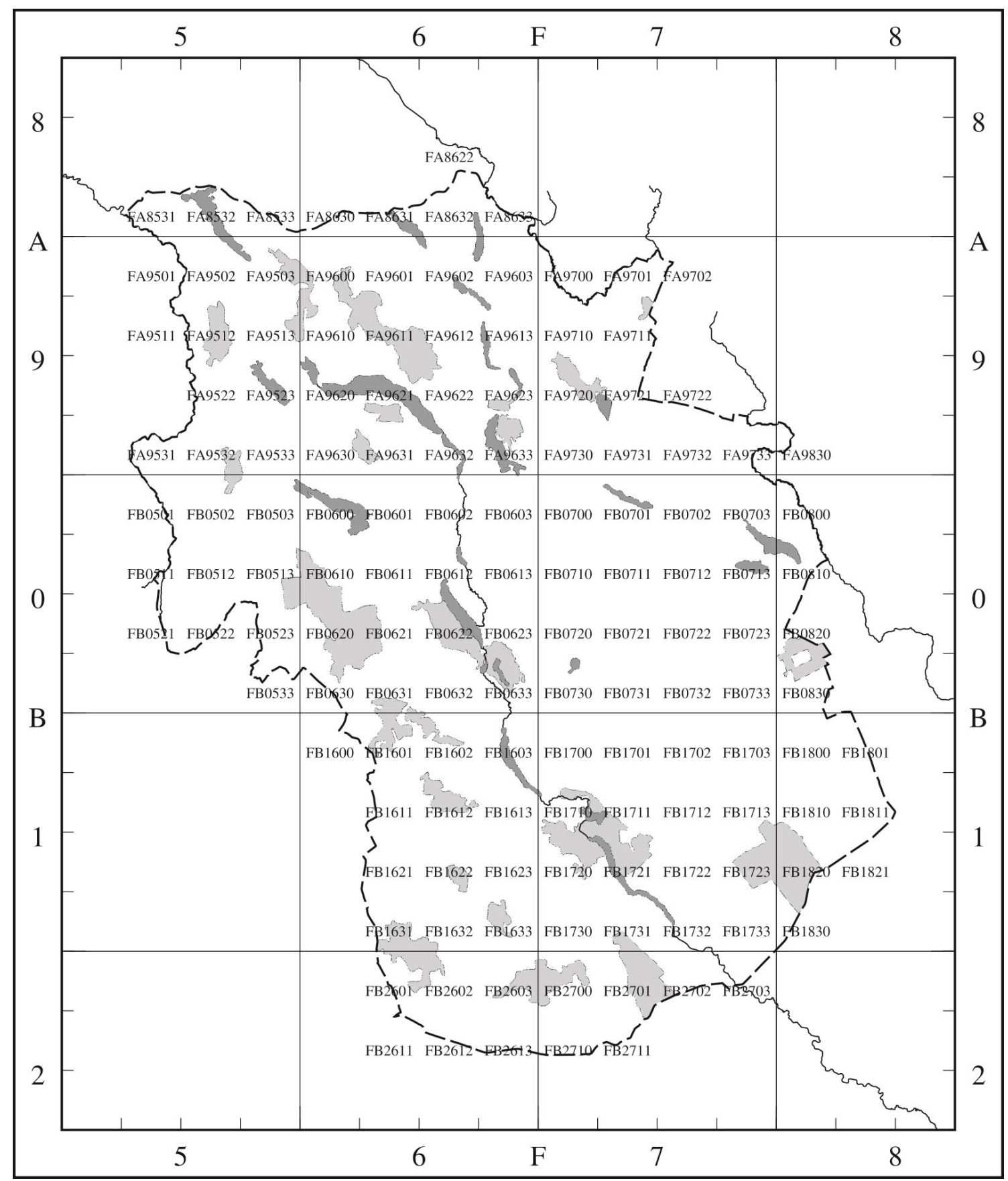

Fig 2: The codes of the ATPOL cartogram units for the study area. Explanations: the capital letters indicate the square of $100 \mathrm{~km}$ side, two numbers in the middle indicate the square of 10-km side, and the last two numbers indicate the square of $2.5 \mathrm{~km}$ side. The boundaries of the study area (dashed line), main rivers, lakes, and forests in the background.

Acknowledgements: I would like to thank Józef Gajda for preparing the maps of study area with the ATPOL cartogram grid.

\section{References}

Abromeit J., Neuhoff W. \& Steffen H. (1898-1940): Flora von Ost- und Westpreussen. Kommisionverlag Gräfe und Unzer, Berlin, Königsberg, 1248 pp.

Ber A. (1981): Pojezierze Suwalsko-Augustowskie. Przewodnik Geologiczny. Wydawnictwa Geologiczne, Warszawa, $192 \mathrm{pp}$. 
Głowacki Z., Falkowski M., Krechowski J., Marciniuk J., Marciniuk P., Nowicka-Falkowska K. \& Wierzba M. (2003): Czerwona lista roślin naczyniowych Niziny Południowopodlaskiej. - Chrońmy Przyr. Ojcz. 59(2): 5-41.

IUCN Standards and Petitions Subcommittee (2016): Guidelines for using the IUCN red list categories and criteria version 12 (February 2016). Prepared by the Standards and Petitions Subcommittee. Downloaded from http://www.iucnredlist.org/documents/RedListGuidelines.pdf

Jackowiak B., Celka Z., Chmiel J., Latowski K. \& Żukowski W. (2007): Red list of vascular flora of Wielkopolska (Poland). - Biodiv. Res. Conserv. 5-8: 95-127.

Jakubowska-Gabara J. \& Kucharski L. (1999): Ginące i zagrożone gatunki flory naczyniowej zbiorowisk naturalnych i półnaturalnych Polski Środkowej. - Fragm. Florist. Geobot. Polon., 6: 55-74.

Kaźmierczakowa R., Bloch-Orłowska J., Celka Z., Cwener A., Dajdok Z., Michalska-Hejduk D., Pawlikowski P., Szczęśniak E. \& Ziarnek K. (2016): Polska czerwona lista paprotników i roślin kwiatowych. Polish red list of pteridophytes and flowering plants. Instytut Ochrony Przyrody Polskiej Akademii Nauk, Kraków, 44 pp.

Kącki Z., Dajdok Z. \& Szczęśniak E. (2003): Czerwona lista roślin naczyniowych Dolnego Śląska. 9-65 pp. In: Kącki Z. (ed): Zagrożone gatunki flory naczyniowej Dolnego Śląska. Instytut Biologii Roślin, Uniwersytet Wrocławski, Polskie Towarzystwo Przyjaciół Przyrody ,pro Natura”, Wrocław, 245 pp.

Kondracki J. (1994): Geografia Polski. Mezoregiony fizyczno-geograficzne. Wydawnictwo Naukowe PWN, Warszawa, $339 \mathrm{pp}$.

Lorenc H. (ed.) (2005): Atlas klimatu Polski. Instytut Meteorologii i Gospodarki Wodnej, Warszawa, 116 pp.

Markowski R. \& Buliński M. (2004): Ginące i zagrożone rośliny naczyniowe Pomorza Gdańskiego. - Acta Bot. Cassubica, Monogr. 1: 1-75.

Mirek Z., Piękoś-Mirkowa H., Zając A. \& Zając M. (2002): Flowering plants and pteridophytes of Poland, a checklist. W. Szafer Institute of Botany, Polish Academy of Sciences, Kraków, 442 pp.

Pliszko A. (2012): Przemiany antropogeniczne flory i bioróżnorodność roślin naczyniowych Pojezierza Zachodniosuwalskiego. Praca doktorska, Instytut Botaniki, Uniwersytet Jagielloński, Kraków, $244+107$ pp. (manuscript of $\mathrm{PhD}$ thesis)

- (2014a): Flora roślin naczyniowych Pojezierza Zachodniosuwalskiego. - Prace Botaniczne 48: 1-349.

- (2014b): Nasięźrzał pospolity Ophioglossum vulgatum w dolinie górnej Rospudy. - Chrońmy Przyr. Ojcz. 70(4): 355-357.

- (2015): New floristic records from the Polish part of the Lithuanian Lakeland (NE Poland). - Steciana 19(1): 25-32.

- (2016): Additions to vascular plant flora of the Western Suwałki Lakeland, north-eastern Poland. - Bot. Lithuanica 22(2): 178-181.

Rutkowski L. (2004): Klucz do oznaczania roślin naczyniowych Polski niżowej. Wydawnictwo Naukowe PWN, Warszawa, 814 pp.

Zając A. (1978): Atlas of distribution of vascular plants in Poland (ATPOL). - Taxon 27(5-6): 481-484.

Zając A. \& Zając M. (eds) (2001): Distribution atlas of vascular plants in Poland. Laboratory of Computer Chorology, Institute of Botany, Jagiellonian University, Kraków, 714 pp.

- (2009): The geographical elements of native flora of Poland. Laboratory of Computer Chorology, Institute of Botany, Jagiellonian University, Kraków, 94 pp.

Zarzycki K. (1986): Lista wymierających i zagrożonych roślin naczyniowych Polski. 11-27 pp. In: Zarzycki K. \& Wojewoda W. (eds): Lista roślin wymierających i zagrożonych w Polsce. Państwowe Wydawnictwo Naukowe, Warszawa, 128 pp.

Zarzycki K. \& Szeląg Z. (1992): Czerwona lista roślin naczyniowych zagrożonych w Polsce. 87-98 pp. In: Zarzycki K., Wojewoda W. \& Heinrich Z. (eds): Lista roślin zagrożonych w Polsce. Wyd. 2. Instytut Botaniki im. W. Szafera PAN, Kraków, 98 pp.

Zarzycki K. \& Szeląg Z. (2006): Red list of the vascular plants in Poland. 9-20 pp. In: Mirek Z., Zarzycki K., Wojewoda W. \& Szeląg Z. (eds): Red list of plants and fungi in Poland. W. Szafer Institute of Botany, Polish Academy of Sciences, Kraków, 99 pp.

Author's address: Artur Plis zko, Department of Taxonomy, Phytogeography and Paleobotany, Institute of Botany, Jagiellonian University in Kraków, Kopernika 31, 31-501 Kraków, Poland. E-mail: artur.pliszko@uj.edu.pl 